\title{
Large chondrosarcoma of the rib invading the mediastinum and the spine
}

Hideo Morioka, MD, PhD, ${ }^{a}$ Hiroo Yabe, MD, PhD, ${ }^{a}$ Shinjiro Kaneko, MD, PhD, ${ }^{a}$ Hironari Takaishi, MD, PhD,

Toshihiko Ueda, MD, PhD, ${ }^{\mathrm{b}}$ Masazumi Watanabe, MD, PhD, ${ }^{\mathrm{c}}$ Koichi Kobayashi, MD, PhD, ${ }^{\mathrm{c}}$ and

Yoshiaki Toyama, MD, PhD, ${ }^{a}$ Tokyo and Utsunomiya, Japan

$\mathrm{W}$

ide excision, the principal treatment for chondrosarcoma, is difficult in the case of large tumors originating from the trunk. The operation sometimes becomes nearly impossible when surrounding important organs are also infiltrated. In this article, we report the case history of a patient with an extremely large chondrosarcoma originating from the first rib and invading the mediastinum and the spine.

\section{Clinical Summary}

A 47-year-old man had a 20-year history of a hard mass on the left side of the neck. Chondrosarcoma arising from the left first rib had been diagnosed at a university hospital where he underwent his first operation for the tumor. Thereafter, the mass recurred, and he had visited several medical institutions for neurologic symptoms in the left arm and dyspnea on exertion. However, successful resection of the tumor was judged to be impossible because of its huge size and infiltration into the thoracic cavity, mediastinum, and spine.

On admission to our hospital, blood pressure was normal. However, he was dyspneic and chest auscultation revealed diminished breath sounds over the left lung regions. His head was tilted about $30^{\circ}$ to the right and a hard tumor was palpable on the left side of his neck and left shoulder joint, with marked restriction of the range of motion of the cervical spine. In addition, his left hand distal to the wrist joint was cold to the touch and the radial and ulnar arterial pulses were not palpable. Pulmonary function testing revealed a reduction of the percent vital capacity to $57 \%$. A plain $\mathrm{x}$-ray film of the chest revealed elevation of the left hemidiaphragm and a large opacity with an ill-defined border on the left side of his neck and left shoulder joint measuring about $19 \times 15 \mathrm{~cm}$. The opacity was not homogeneous and showed conspicuous calcification. On chest computed tomography, the tumor was found to extend from the

\footnotetext{
From the Department of Orthopaedic Surgery, School of Medicine, Keio University, Tokyo, Japan, ${ }^{a}$ the Department of Cardiovascular Surgery, Saiseikai Utsunomiya Hospital, Utsunomiya, Japan, ${ }^{\mathrm{b}}$ the Department of Thoracic Surgery, School of Medicine, Keio University, Tokyo, Japan. ${ }^{c}$

Received for publication May 14, 2006; accepted for publication June 15, 2006.

Address for reprints: Hideo Morioka, MD, PhD, Department of Orthopaedic Surgery, School of Medicine, Keio University, 35 Shinanomachi, Shinjuku-ku, Tokyo 160-8582, Japan (E-mail: morioka@sc.itc.keio.ac.jp).

J Thorac Cardiovasc Surg 2006;132:986-7

$0022-5223 / \$ 32.00$

Copyright $\odot 2006$ by The American Association for Thoracic Surgery doi:10.1016/j.jtcvs.2006.06.032
}

thoracic cavity to the mediastinum and the spine, in contact with the aortic arch and the left carotid vessels, and to invade the spine in the midline region. On magnetic resonance imaging, the tumor showed low to iso-intensity on T1-weighted images and irregularly mixed low to high intensity on T2-weighted images (Figure 1). After injection of gadolinium, T1-weighted images showed enhancement of the regions surrounding the tumor and spots within the tumor. On angiography, the left subclavian artery was blocked several centimeters from its origin and an indentation was noted in the aorta (Figure 2).

The operation was performed by thoracotomy with resection of the left clavicle and the left second rib. The tumor was first sectioned and then removed while neurovascular bundles, which were compressed downward, were preserved. The lesion invading the spine was curetted. Histopathologic examination of the resected specimens confirmed the diagnosis of chondrosarcoma (grade 1 to 2). Although pleural effusion developed and dyspnea worsened postoperatively, both were transient and the patient gradually recovered. Local recurrence developed in the spine 4 years after the operation, and was resected. At present, 5 years after the operation, the patient is alive without any evidence of recurrence.

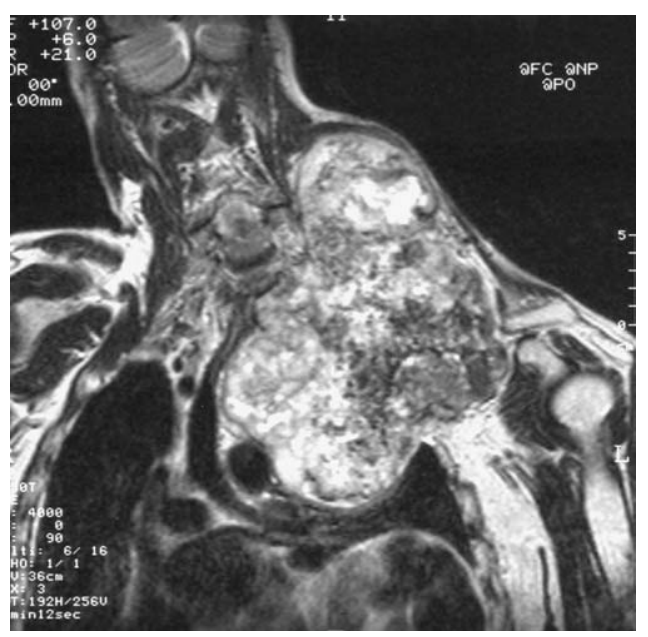

Figure 1. On magnetic resonance imaging, the tumor showed irregularly mixed low to high intensity on T2-weighted images. It extended from the thoracic cavity to the mediastinum and the spine, in contact with the aortic arch and the left carotid vessels. 


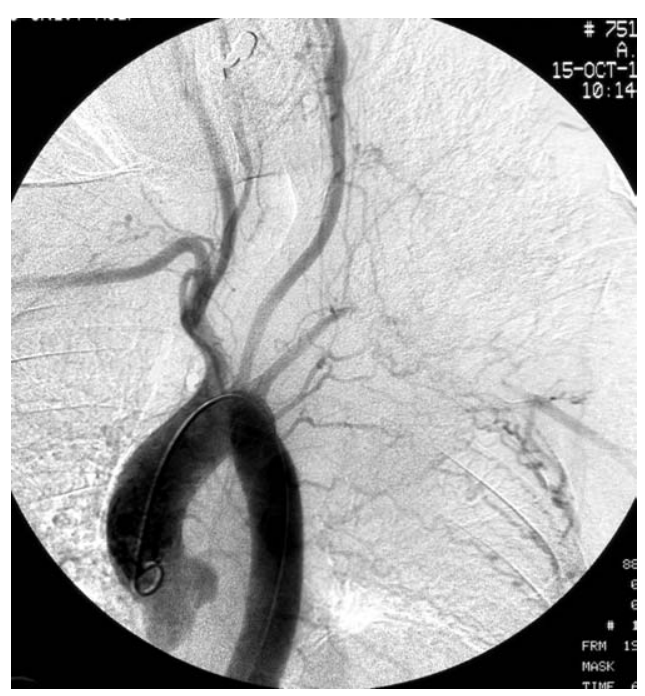

Figure 2. Angiography showed that the left subclavian artery was blocked several centimeters from its origin and an indentation was noted in the aorta.

\section{Discussion}

Current therapy for chondrosarcoma requires adequate surgical excision, because radiation therapy and chemotherapy have not been shown to be effective. ${ }^{1}$ On the other hand, large chondrosarcomas of the chest wall are difficult lesions to treat because of the anatomic proximity of vital neurovascular structures and the limited surgical margins that can be achieved. Intralesional surgery for chondrosarcoma is an inadequate procedure; however, sometimes it cannot be avoided in the case of a large tumor originating from the trunk, such as the chest wall or pelvis. ${ }^{2}$ With regard to prognostic factors, both histologically high-grade malignancy and origin from the trunk are known to be risk factors for local recurrence. ${ }^{1}$ However, only the former is considered to affect survival directly. ${ }^{3,4}$ Ozaki and associates ${ }^{5}$ also concluded that intralesional surgery for chondrosarcoma often leads to local recurrence, but it does not always result in metastases and death. This suggests that a good prognosis in terms of survival can be expected, even in cases in which adequate wide resection cannot be achieved, so long as the histologic malignancy grade is low. It is important to plan the treatment strategy for chondrosarcomas, which can occasionally become huge, taking into consideration not only the size and anatomic location of the tumor, but also the histologic malignancy grade of the tumor.

\section{References}

1. Lee FY, Mankin HJ, Fondren G, Gebhardt MC, Springfield DS, Rosenberg AE, et al. Chondrosarcoma of bone: an assessment of outcome. J Bone Joint Surg. 1999;81 A:326-38.

2. Pring ME, Weber KL, Unni KK, Sim FH. Chondrosarcoma of the pelvis. A review of sixty-four cases. J Bone Joint Surg. 2001;83A: 1630-42.

3. Evans HL, Ayala AG, Romsdahl MM. Prognostic factors in chondrosarcoma of bone: A clinicopathologic analysis with emphasis on histologic grading. Cancer. 1977;40:818-31.

4. Fiorenza F, Abudu A, Grimer RJ, Carter SR, Tillman RM, Ayoub K, et al. Risk factors for survival and local control in chondrosarcoma of bone. J Bone Joint Surg. 2002;84B:93-9.

5. Ozaki T, Lindner N, Hillmann A, Rodl R, Blasius S, Winkelmann W. Influence of intralesional surgery on treatment outcome of chondrosarcoma. Cancer. 1996;77:1292-7. 